Check for updates

Cite this: RSC Adv., 2017, 7, 28024

\title{
Poly(2-vinylpyridine) brushes as a reaction chamber to fabricate spiky gold nanoparticles $\dagger$
}

\author{
Wenqin Wang, (D) *a Hanzhi Zou, ${ }^{a}$ Guoke Xing, ${ }^{a}$ Mengying Shang ${ }^{a}$ and Tao Chen (D) ${ }^{b}$
}

Using polymer brushes to guide the growth of gold nanostructures is of great scientific and technological importance. We report a new strategy to tune the shape of gold nanoparticles (NPs) on poly(2-vinylpyridine) (P2VP) brushes through vapor deposition polymerization of pyrrole: P2VP brushes loaded with $\mathrm{AuCl}_{4}{ }^{-}$ions were exposed to pyrrole vapor, one-step synthesized gold NPs and conducting polymer polypyrrole (PPy). The spiky gold NPs were obtained by controlling the thickness of P2VP brushes and reaction time, and a possible mechanism was proposed. The as-obtained spiky gold NPs could be used as substrates for surface-enhanced Raman scattering (SERS). They exhibited excellent Raman enhancement performance and the enhancement factor (EF) was in the range $(4.0-6.2) \times 10^{5}$ when 4 -aminothiophenol was used as a probe molecule.

Received 10th March 2017

Accepted 22nd May 2017

DOI: $10.1039 / \mathrm{c} 7 \mathrm{ra02906c}$

rsc.li/rsc-advances
It is well known that one of the main influence factors for morphology of noble metal particles is kinetic controlling. ${ }^{\mathbf{1 2 - 1 4}}$ Previous reports have demonstrated that a small change of growth rates of individual metal crystal facets could result in drastic change in the morphology and size of resultant metal crystalline structure. ${ }^{13}$ Therefore, to metal NPs/polymer brushes system, controlling the growth rate of noble metal NPs within polymer brushes and simultaneously hindering original function of polymer brushes (limiting NPs growth and their aggregation) would realize shape-controlled growth of noble metal NPs using polymer brushes as matrices. In this work, we attempted to use heterocyclic aromatic compound-pyrrole as reductant rather than $\mathrm{NaBH}_{4}$ to reduce $\mathrm{AuCl}_{4}{ }^{-}$ions adsorbed on poly(2-vinylpyridine) (P2VP) brushes. Because the standard reduction potential of the $\mathrm{AuCl}_{4}{ }^{-}$is higher than the oxidation potential of pyrrole, pyrrole could be oxidized by $\mathrm{AuCl}_{4}{ }^{-}$to conducting polymer-polypyrrole (PPy) and $\mathrm{AuCl}_{4}{ }^{-}$be reduced to zero-valent gold simultaneously. ${ }^{15}$ In above strategy, besides pyrrole as a relative weaker reducing agent lowed the growth rates of gold crystal, the presence of the formed PPy within polymer brushes could mitigate the polymer brushes' disadvantage of limiting NPs growth, which would contribute to formation of gold NPs with complex morphology. Our strategy is schematically shown in Scheme 1: incubating P2VP brushes

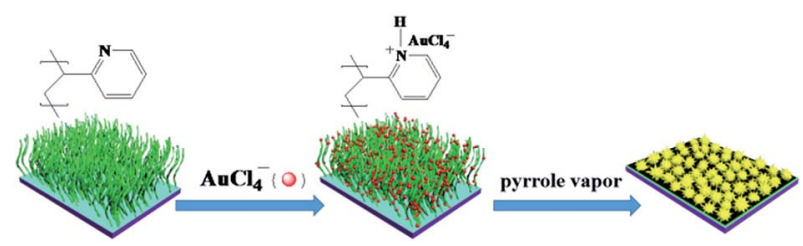

Scheme 1 Schematic illustration of the fabrication of gold-PPy nanocomposites on P2VP brushes. 
with $\mathrm{HAuCl}_{4}$ aqueous solution, the pyridyl groups, present along the polymer chains, were highly protonated and $\mathrm{AuCl}_{4}{ }^{-}$ ions were bound tightly to the P2VP chains as counter ions of the protonated pyridine groups. When P2VP brushes loading $\mathrm{AuCl}_{4}{ }^{-}$ions were exposed to pyrrole vapors, $\mathrm{AuCl}_{4}{ }^{-}$ions acted both as an oxidant for pyrrole monomer and as a source of $\mathrm{Au}$ atoms, one-step yielding gold-PPy nanocomposites on P2VP brushes. By controlling the thickness of P2VP brushes and reaction times, gold nanostructures with different morphology were obtained.

\section{Experimental section}

\subsection{Materials}

2-Vinylpyridine, 3-aminopropyltriethoxysilane (APTES), and 4aminothiophenol were purchased from Sigma. Pyrrole, $\mathrm{HAuCl}_{4} \cdot 4 \mathrm{H}_{2} \mathrm{O}(99.8 \%)$, toluene and $\mathrm{H}_{2} \mathrm{O}_{2}(25 \%)$ were obtained from Sinopharm Chemical Reagent Co. (Shanghai, China). 2Vinylpyridine was purified by neutral $\mathrm{Al}_{2} \mathrm{O}_{3}$ column and pyrrole was distilled under reduced pressure before use. Other reagents were used as received without further purification. Highly polished single-crystal silicon wafers (N-doped, (100)oriented silicon with a $\mathrm{SiO}_{2}$ layer thickness of $200 \mathrm{~nm}$ ) were cleaned with "piranha" solution (Caution: Piranha solution reacts violently with organic matter) at $80{ }^{\circ} \mathrm{C}$ for $2 \mathrm{~h}$, subsequently washed thoroughly with deionized water, and dried in a stream of $\mathrm{N}_{2}$.

\subsection{Surface-initiated polymerization by photografting and photopolymerization (SIPGP)}

SIPGP technology according to previous reports: ${ }^{1,16,17}$ A silicon wafer substrate which was treated with fresh "piranha" solution was immersed into a 5\% APTES anhydrous toluene solution and sonicated for $30 \mathrm{~min}$. Then, APTES molecules were attached as linker molecules via a silanization reaction. After thoroughly cleaned and dried by $\mathrm{N}_{2}$, the APTES-functionalized silicon wafer substrate was submerged in bulk 2-vinylpyridine and irradiated for 3 or $5 \mathrm{~h}$ with an UV-light lamp with the spectral distribution between 300 and $400 \mathrm{~nm}\left(\lambda_{\max }=350 \mathrm{~nm}\right)$ for the SIPGP. After the photopolymerization, the substrate was rigorously rinsed with different organic solvents in order to remove physisorbed polymer.

\subsection{Preparation of gold-PPy composites on P2VP brushes}

The P2VP brushes were incubated overnight in an aqueous solution of $\mathrm{HAuCl}_{4}(1 \mathrm{wt} \%)$. P2VP brushes loading $\mathrm{AuCl}_{4}{ }^{-}$ions were subsequently rinsed with deionized water and put into a reaction vessel $(50 \mathrm{~mL})$ equipped with a pyrrole solution loading reservoirs. The monomer in the reservoirs evaporated gradually and diffused into P2VP brushes loading $\mathrm{AuCl}_{4}{ }^{-}$ions, where the reaction was occurred. After reaction for a certain period (15 $\mathrm{min}, 30 \mathrm{~min}$, and $1 \mathrm{~h}$ ), the obtained products were rinsed with ethanol and dried in a vacuum oven at $50{ }^{\circ} \mathrm{C}$.

\subsection{Preparation of samples for surface-enhanced Raman scattering}

The as-obtained gold NPs on silicon wafer were immersed into 4-aminothiophenol (4-ATP) ethanol solution with different concentrations for $3 \mathrm{~h}$. Subsequently, the samples were rinsed with ethanol, and dried under nitrogen for SERS measurement.

\subsection{Characterization}

The morphology of the samples was observed by Scanning electron microscope (SEM, SU-70). X-ray photoelectron spectroscopy (XPS, Axis Ultra dld) was used to characterize chemical information of the products. Thermogravimetric analysis was determined with a PerkineElmer thermogravimetric analyzer (TG-DTA, SSC-5200) at a heating rate of $10^{\circ} \mathrm{C} \mathrm{min}^{-1}$ from room temperature up to $850^{\circ} \mathrm{C}$ under air atmosphere. Raman spectra were recorded by using a microscopic confocal Raman spectrometer (Renishaw PLC) employing a $785 \mathrm{~nm}$ laser beam and acquisition time of $30 \mathrm{~s}$.

\section{Results and discussion}

In our work, the P2VP brushes with different thickness $(40 \mathrm{~nm}$ and $75 \mathrm{~nm}$ ) were fabricated by SIPGP technology (the thickness of P2VP brushes was determined by atomic force microscopy (AFM), as shown in Fig. S1, ESI $\dagger$ ). Grafting density of the obtained P2VP (75 nm thickness) was estimated to as 0.74 chains per $\mathrm{nm}^{2}$ by $\sigma=1 / d_{\mathrm{g}}{ }^{2}$, where $d_{\mathrm{g}}$ is the distance between two grafting sites, which was derived from $d_{\mathrm{g}}=M_{\mathrm{n}}{ }^{1 / 2}\left(N_{\mathrm{A}} d \rho\right)^{-1 / 2}$, where $M_{\mathrm{n}}\left(72000 \mathrm{~g} \mathrm{~mol}^{-1}\right)$ is the number average molecular weight of polymer chains, $N_{\mathrm{A}}$ is the Avogadro's number, and $\rho\left(1.18 \mathrm{~g} \mathrm{~cm}^{-3}\right)$ is the density of P2VP. ${ }^{18}$ Because $\mathrm{dg}$ was calculated as $1.16 \mathrm{~nm}$, which is smaller that the radius of gyration of P2VP polymer coils $\left(R_{\mathrm{g}} \sim 5 \mathrm{~nm}\right)$; so, the polymer layer can be considered as polymer brushes. ${ }^{19}$

Fig. 1 shows the growth of the gold-PPy nanostructures when P2VP brushes (40 nm thickness) loading $\mathrm{AuCl}_{4}{ }^{-}$ions were exposed to pyrrole vapor for different time $(15 \mathrm{~min}, 30 \mathrm{~min}$, and $1 \mathrm{~h}$ ). In the initial stage $(15 \mathrm{~min})$, some gold nanoclusters are obtained (Fig. 1(A, a)). With reaction time prolongs to $30 \mathrm{~min}$, flower-like gold nanoclusters are exhibited in Fig. $1(\mathrm{~B}, \mathrm{~b})$. When reaction time increases up to $1 \mathrm{~h}$, some raspberry-like gold nanostructures decorated on bumpy PPy layer are observed (Fig. 1(C, c)).

When P2VP brushes (75 $\mathrm{nm}$ thickness) loading $\mathrm{AuCl}_{4}{ }^{-}$ions were exposed to pyrrole vapors for different time $(15 \mathrm{~min}$, $30 \mathrm{~min}$, and $1 \mathrm{~h}$ ), the SEM images of the as-obtained gold-PPy composites are shown in Fig. 2. With reaction time prolongs, nanoclusters (Fig. 2(A, a)), spherical nanostructures (Fig. 2(B, b)), and spinous nanostructures (Fig. 2(C, c)) are observed, respectively. It is noted that the lower half of each spiky spherical NP is embedded into a thick PPy layer (Fig. 2c).

The actual formation mechanism is, as of now, unclear, a hypothesis is proposed. In our work, pyrrole is a relatively weaker reducing agent compared to $\mathrm{NaBH}_{4}$. Pyrrole evaporates slowly to form vapor, which diffuses gradually to contact $\mathrm{AuCl}_{4}{ }^{-}$ ions adsorbed on P2VP chains. Therefore, the reaction rate of 


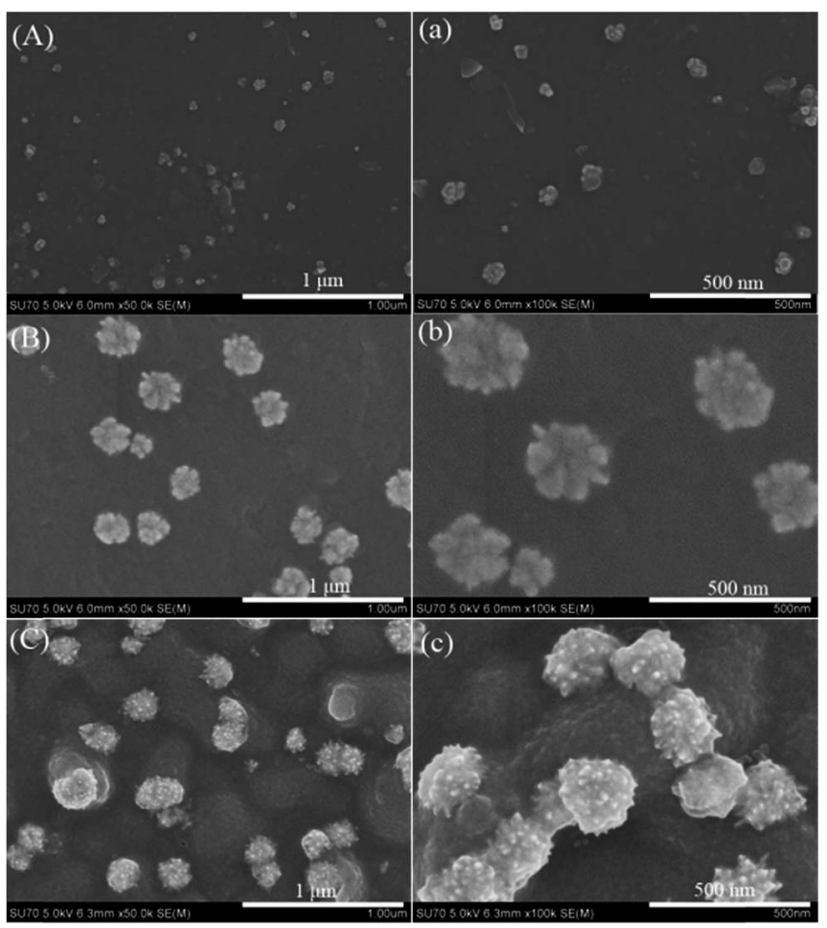

Fig. 1 Low- and high-magnification SEM images of the gold-PPy nanostructures when P2VP brushes (40 nm thickness) loading $\mathrm{AuCl}_{4}{ }^{-}$ions exposed to pyrrole vapors for (A, a) $15 \mathrm{~min},(B, b) 30 \mathrm{~min}$, and (C, c) $1 \mathrm{~h}$.

pyrrole oxidized by $\mathrm{AuCl}_{4}{ }^{-}$ions is very slow. Initially, tiny gold nanocrystal and pyrrole oligomers are formed gradually along each P2VP chain. According to the report by Kim, sufficiently

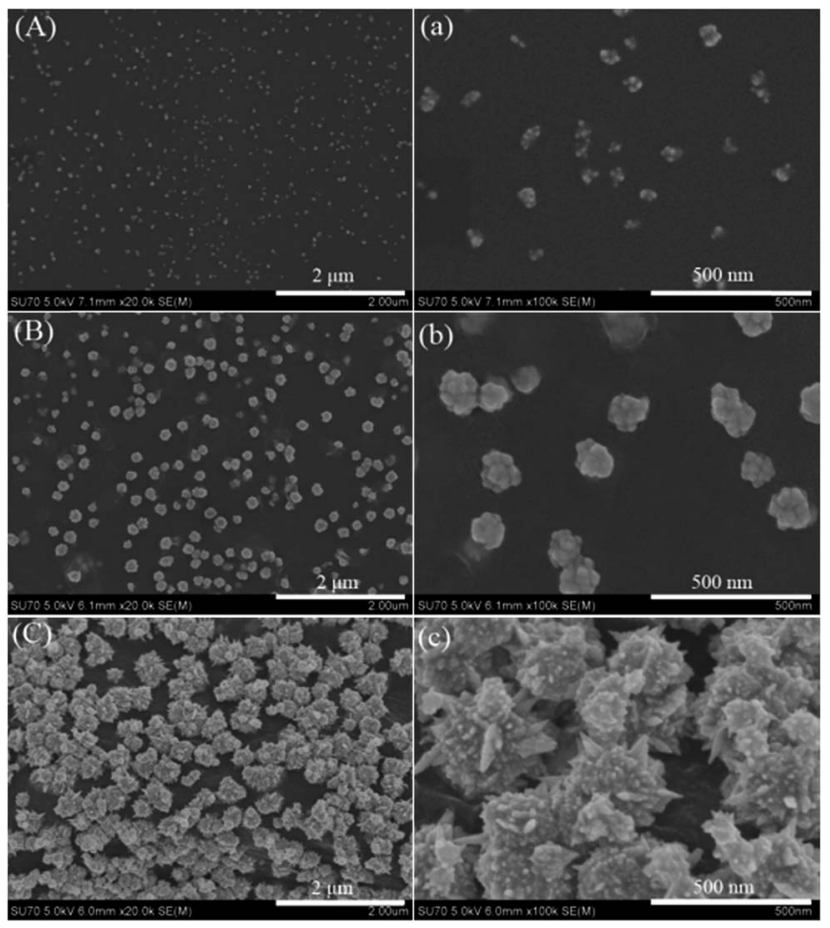

Fig. 2 Low- and high-magnification SEM images of the gold-PPy composites when P2VP brushes (75 nm thickness) loading $\mathrm{AuCl}_{4}^{-}$ions exposed to pyrrole vapors for (A, a) $15 \mathrm{~min},(B, b) 30 \mathrm{~min}$, and $(C, C) 1 \mathrm{~h}$. small NPs can disperse freely within the polymer brushes. ${ }^{20}$ Thus, in this case, those tiny gold nanocrystal and pyrrole oligomers can be immobilized within P2VP brushes. However, as the reaction continues, those gold nanocrystal and pyrrole oligomers grow increasingly into larger NPs, which are moved toward the polymer brush surface due to repulsive force imposed by the polymer chains. ${ }^{21}$ When all P2VP chains are wrapped completely by the composites of gold nanocrystal and PPy, the P2VP chains can not work well for preventing aggregation, which results in gold-PPy composites layer on the surface P2VP brushes. In addition, it is also found that the final gold NPs existed on the surface of PPy layer instead of being embedded into PPy layer, which may be attributed to PPy growth in lateral directions (parallel to substrate) and gold NPs growth towards PPy-air interface. As to the significant morphology difference of gold NPs shown in Fig. 1 and 2, it could be interpreted that the amount of $\mathrm{AuCl}_{4}{ }^{-}$ions loaded by thicker P2VP brushes is more than that of thinner P2VP brushes, which provides sufficient source of gold for forming complex, hierarchical gold nanostructures.

To determine the surface composition, X-ray photoelectron spectrum (XPS) was employed to analyze the relevant samples. Fig. 3A shows the wide scan spectra of pure P2VP brushes and gold-PPy/P2VP composites. Compared with XPS spectrum of pure P2VP brushes, besides $\mathrm{C} 1 \mathrm{~s}, \mathrm{~N} 1 \mathrm{~s}$ and $\mathrm{O} 1 \mathrm{~s}, \mathrm{Au} 4 \mathrm{f}$ is observed. In high-resolution Au $4 \mathrm{f}$ XPS spectrum (Fig. 3B), the peak of $\mathrm{Au} 4 \mathrm{f}$ can be flitted into a main doublet peaks, and signals appeared at about $87.4 \mathrm{eV}$ and $83.6 \mathrm{eV}$ are assigned to $\mathrm{Au}$ $4 \mathrm{f}_{5 / 2}$ and $\mathrm{Au} 4 \mathrm{f}_{7 / 2}$, respectively, ${ }^{3}$ indicating the $\mathrm{AuCl}_{4}{ }^{-}$ions have been reduced to zero-valent gold $\left(\mathrm{Au}^{0}\right)$. The $\mathrm{N} 1 \mathrm{~s}$ high-resolution spectrum of pure P2VP brushes and gold-PPy/P2VP composites is shown in Fig. $2 \mathrm{C}$ and $\mathrm{D}$, respectively. The $\mathrm{N} 1 \mathrm{~s}$ peak at $399.3 \mathrm{eV}$ is the characteristic peak of P2VP. ${ }^{22}$ Compared with pure P2VP brushes, it is also found that the $\mathrm{N} 1 \mathrm{~s}$ signal of goldPPy/P2VP composites is slightly shifted to higher energy, which is indicative for the formation of PPy. The peak-fitted $\mathrm{N} 1 \mathrm{~s}$ coreline spectrum of the gold-PPy/P2VP composites (Fig. 3D) shows that two nitrogen environments are present. The peaks at $399.9 \mathrm{eV}$ is attributed to neutral nitrogen atoms (-NH-) in PPy. The peak at $401.4 \mathrm{eV}$ is assigned to positively charged nitrogen $\left(-\mathrm{N}^{+}-\right)$species in PPy. ${ }^{23}$ The TGA of gold-PPy composites is shown in Fig. S2 (ESI $\dagger$ ).

It is known that the dramatic Raman signal enhancement occurs on "hot spots", which locate in the sharp corners and edges of noble metal nanostructures, or nanoscale junctions between two adjacent noble metal NPs. ${ }^{8-11}$ Therefore, the spiky gold NPs (shown in Fig. 2C) is an ideal SERS substrate. Fig. 4 shows that SERS spectra of the spiky gold NPs using 4-ATP as the target analyte. Compared to the spectrum of the solid 4-ATP, the significant differences in the SERS of the gold NPs are the frequency shift for some bands and changes in the relative intensity. The $v_{\mathrm{cs}}$ band shifts from 1085 to $1079 \mathrm{~cm}^{-1}$ and $v_{\mathrm{cc}}$ band shifts from 1594 to $1588 \mathrm{~cm}^{-1}$, indicating the -SH group in 4-ATP directly contacts the gold surface to form the Au-S bond. ${ }^{24,25}$ To inquire quantitatively the enhancement effect of 4ATP on spiky gold NPs, the surface enhancement factor (EF) value of 4-ATP on gold NPs is calculated according to the 

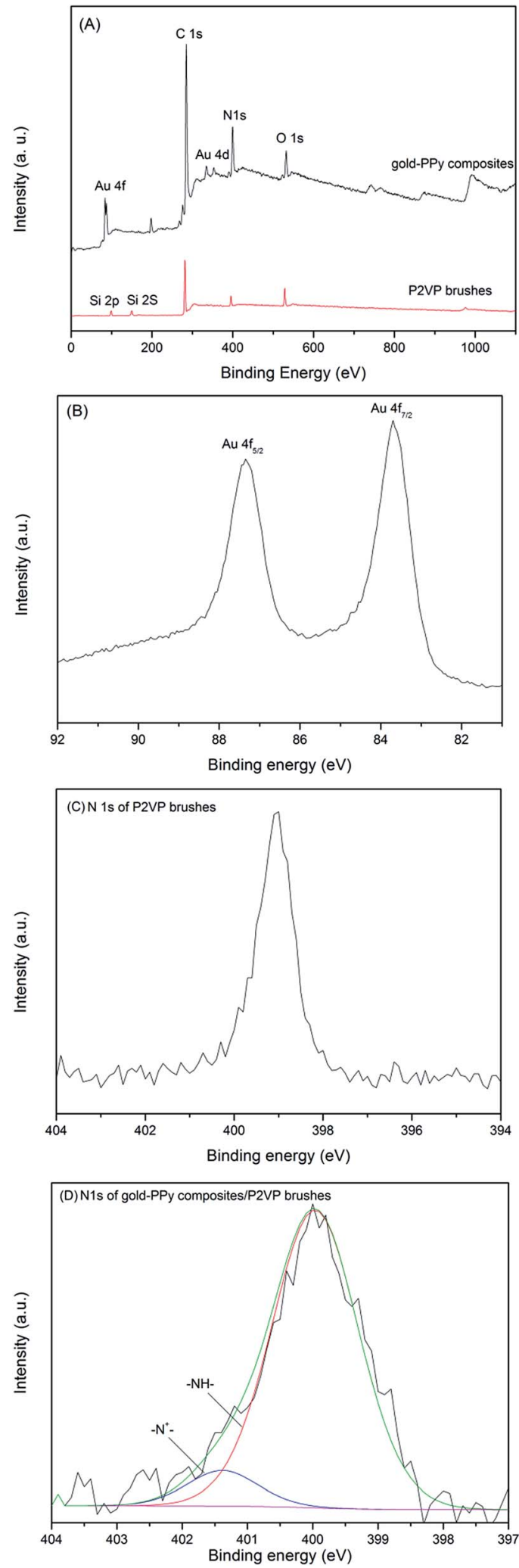

Fig. 3 (A) XPS of the pure P2VP brushes and gold-PPy composites/ P2VP brushes, (B) Au 4f XPS spectrum of gold-PPy composites/P2VP brushes, (C) N 1s XPS spectrum of pure P2VP brushes, and (D) N 1s XPS spectrum of gold-PPy composites/P2VP brushes.

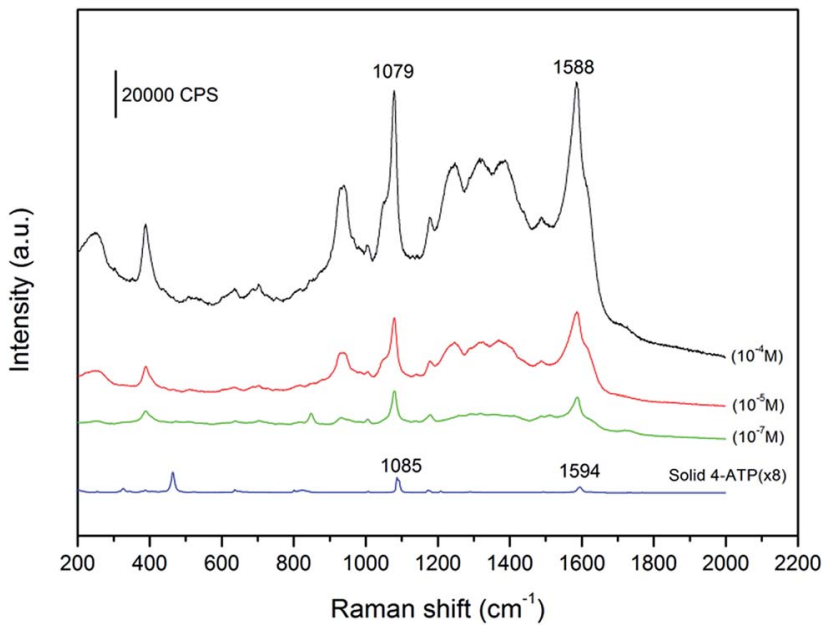

Fig. 4 Surface-enhanced Raman spectra of 4-ATP with different concentrations adsorbed on spiky gold NPs and Raman spectrum of solid 4-ATP.

following expression: $\mathrm{EF}=\left[I_{\mathrm{SERS}}\right] /\left[I_{\mathrm{Ref}}\right] \times\left[N_{\mathrm{Ref}}\right] /\left[N_{\mathrm{ads}}\right],{ }^{26}$ where $I_{\text {SERS }}$ is the intensity of a vibrational mode in the SERS spectrum of 4-ATP and $I_{\text {Ref }}$ is the intensity of the same mode in the Raman spectrum from the solid 4-ATP. For all spectra, the intensity of $v_{\text {cs }}$ at $1079 \mathrm{~cm}^{-1}$ is used to calculate EF values. $N_{\text {Ref }}$ and $N_{\text {ads }}$ are the numbers of SERS molecules in the reference and sample within the laser spot. In our experiment, the focused laser beam with the laser spot ( $1 \mu \mathrm{m}$ in diameter) and the penetration depth $(2 \mu \mathrm{m})$ is used. $N_{\text {Ref }}$ is calculated assuming a cylindrical focal volume (the diameter of the laser spot is $1 \mu \mathrm{m}$ and the depth of the laser is $2 \mu \mathrm{m}$ ). Then, $N_{\text {Ref }}$ of SERS molecules is calculated by $N_{\text {av }}\left(\rho V_{\text {laser }} / M\right)$, in which $N_{\text {av }}$ is the Avogadro number, $V_{\text {laser }}$ is the focal volume of the laser illumination, $M$ is the molecular weight of 4-ATP molecule ( $\left.125.19 \mathrm{~g} \mathrm{~mol}^{-1}\right)$, and $\rho$ is the density of 4-ATP molecule $\left(1.18 \mathrm{~g} \mathrm{~cm}^{-3}\right)$. So, $N_{\text {Ref }}$ is $8.91 \times 10^{9}$ per molecules. The number of the adsorbed molecules is poorly defined due to complex morphology of the gold NPs. In this work, we assume the all 4-ATP molecules are uniformly adsorbed on the gold NPs. This value can be obtained according to the method proposed by Orendorff et al., which is $N_{\mathrm{ads}}=N_{\mathrm{d}^{-}}$ $A_{\text {laser }} A_{\mathrm{N}} / \sigma{ }^{27}$ Where $N_{\mathrm{d}}$ is the number density of the gold nanostructures, $A_{\text {laser }}$ is the area of the focal spot of the laser and can be obtained from the diameter of the laser spot $(1 \mu \mathrm{m}) . A_{\mathrm{N}}$ is the NP's footprint area assuming that the gold NPs are spheres with diameter about $200 \mathrm{~nm} . \sigma$ is the surface area occupied by an adsorbed 4-ATP molecule. $N_{\mathrm{d}}$ and $A_{\mathrm{N}}$ can be obtained from the SEM images in Fig. 2C. According to the report by Kim, each 4ATP molecule occupies $\sim 0.2 \mathrm{~nm}^{2}$ on full coverage of Au, indicating that $\sigma$ can be adopted as $\sim 0.20 \mathrm{~nm}^{2}$ per molecule. ${ }^{24} \mathrm{So}$, $N_{\text {ads }}=2.46 \times 10^{6}$ per molecules. It is noticeable that the calculated error is ascribed to the $N_{\mathrm{d}}$ and the area of the gold NPs because they are approximately calculated according to the SEM images. According to the statistical results of the repeated SERS measurements, the ratio of $I_{\text {SERS }}$ to $I_{\text {Ref }}$ is $(1.41 \pm 0.3) \times$ $10^{2}$, so $\mathrm{EF}$ is calculated to be in the range $(4.0-6.2) \times 10^{5}$. This value is ranged with the highest $\mathrm{EF}$ values that have been 
reported for 4-ATP molecules on gold nanostructures with other shapes. ${ }^{24-30}$

\section{Conclusions}

In summary, using $\mathrm{P} 2 \mathrm{VP}$ brushes loading $\mathrm{AuCl}_{4}^{-}$ions as matrices and pyrrole as reductant, we have successfully demonstrated a new strategy to tune the morphology of gold nanostructures. Spiky gold NPs could act as potential SERSactive substrates for trace detection of organic molecules. This approach enriched the scientific research of polymer brushes and may be extended to produce many other types of noble metal-conducting polymer composites and open new pathways for engineering of noble metal nanostructures with unusual shapes.

\section{Acknowledgements}

This work was sponsored by K. C. Wong Magna Fund in Ningbo University, Ningbo Natural Science Foundation (2016A610054), and the Fund for Science and Technology Innovative Team in Zhejiang Province (2011R50001-03).

\section{References}

1 S. Gupta, M. Agrawal, M. Conrad, N. A. Hutter, P. Olk, F. Simon, L. M. Eng, M. Stamm and R. Jordan, Adv. Funct. Mater., 2010, 20, 1-6.

2 E. M. Benetti, X. F. Sui, S. Zapotoczny and G. J. Vancso, Adv. Funct. Mater., 2010, 20, 939-944.

3 D. Paripovic and H. A. Klok, ACS Appl. Mater. Interfaces, 2011, 3, 910-917.

4 O. Azzaroni, A. A. Brown, N. Cheng, A. Wei, A. M. Jonas and W. T. S. Huck, J. Mater. Chem., 2007, 17, 3433-3439.

5 S. Gupta, P. Uhlmann, M. Agrawal, S. Chapuis, U. Oertel and M. Stamm, Macromolecules, 2008, 41, 2874-2879.

6 A. Calvo, M. C. Fuertes, B. Yameen, F. J. Williams, O. Azzaroni and G. J. A. A. Soler-Illia, Langmuir, 2010, 26, 5559-5567.

7 G. K. Nie, G. Z. Li, L. Wang and X. W. Zhang, Polym. Chem., 2016, 7, 753-769.

8 C. Y. Song, N. Zhou, B. Y. Yang, Y. J. Yang and L. H. Wang, Nanoscale, 2015, 7, 17004-17011.

9 Y. J. Huang, A. Dandapat and D.-H. Kim, Nanoscale, 2014, 6, 6478-6481.
10 J. X. Fang, S. Y. Du, S. Lebedkin, Z. Y. Li, R. Kruk, M. Kappes and H. Hahn, Nano Lett., 2010, 10, 5006-5013.

11 G. W. Lu, C. Li and G. Q. Shi, Chem. Mater., 2007, 19, 34333440.

12 M. L. Personick and C. A. Mirkin, J. Am. Chem. Soc., 2013, 135, 18238-18247.

13 T. T. Nhung and S.-W. Lee, ACS Appl. Mater. Interfaces, 2014, 6, 21335-21345.

14 Y. N. Xia, Y. J. Xiong, B. K. Lim and S. E. Skrabalak, Angew. Chem., Int. Ed., 2009, 48, 60-103.

15 S. T. Selvan, J. P. Spatz, H.-A. Klok and M. Möller, Adv. Mater., 1998, 10, 132-134.

16 M. Steenackers, S. Q. Lud, M. Niedermeier, P. Bruno, D. M. Gruen, P. Feulner, M. Stutzmann, J. A. Garrido and R. Jordan, J. Am. Chem. Soc., 2007, 129, 15655-15661.

17 M. Steenackers, A. M. Gigler, N. Zhang, F. Deubel, M. Seifert, L. H. Hess, C. H. Y. X. Lim, K. P. Loh, J. A. Garrido, R. Jordan, M. Stutzmann and I. D. Sharp, J. Am. Chem. Soc., 2011, 133, 10490-10498.

18 R. Contreras-Caceres, C. Dawson, P. Formanek, D. Fischer, F. Simon, A. Janke, P. Uhlmann and M. Stamm, Chem. Mater., 2013, 25, 158-169.

19 B. Zdyrko, V. Klep and I. Luzinov, Langmuir, 2003, 19, 1017910187.

20 J. U. Kim and B. O'Shaughnessy, Phys. Rev. Lett., 2002, 89, 238301.

21 J. U. Kim and B. O'Shaughnessy, Macromolecules, 2006, 39, 413-425.

22 L. Cui and Y. C. Han, Langmuir, 2005, 21, 11085-11091.

23 K. G. Neoh, K. K. S. Lau, V. V. T. Wong and E. T. Kang, Chem. Mater., 1996, 8, 167-172.

24 K. Kim and J. K. Yoon, J. Phys. Chem. B, 2005, 109, 2073120736.

25 Y. L. Wang, S. J. Guo, H. J. Chen and E. K. Wang, J. Colloid Interface Sci., 2008, 318, 82-87.

26 X. G. Hu, T. Wang, L. Wang and S. J. Dong, J. Phys. Chem. C, 2007, 111, 6962-6969.

27 C. J. Orendorff, A. Gole, T. K. Sau and C. J. Murphy, Anal. Chem., 2005, 77, 3261-3266.

28 W. J. Lee, S. Y. Lee, R. M. Briber and O. Rabin, Adv. Funct. Mater., 2011, 21, 3424-3429.

29 S. J. Guo, L. Wang and E. K. Wang, Chem. Commun., 2007, 3163-3165.

30 J. Ye, M. Shioi, K. Lodewijks, L. Lagae, T. Kawamura and P. V. Dorpe, Appl. Phys. Lett., 2010, 97, 163106. 